\title{
Radiotherapy as Prophylactic or Sole Treatment of Heterotopic Ossifications - About Two Case Reports
}

\author{
Andreia Ponte*, Luísa Rolim, António Silva, Tânia Teixeira and Margarida Borrego \\ Radiation Oncology Department, University Hospital of Coimbra, Portugal \\ *Corresponding author: Andreia Ponte, Radiation Oncology Department, University Hospital of Coimbra, Portugal
}

\section{ARTICLE INFO}

Received: 悪 March 23, 2019

Published: 幽 April 04, 2019

Citation: Andreia Ponte, Luísa Rolim, António Silva, Tânia Teixeira and Margarida Borrego. Radiotherapy as Prophylactic or Sole Treatment of Heterotopic Ossifications - About Two Case Reports. Biomed J Sci \& Tech Res 16(5)2019. BJSTR. MS.ID.002907.

\author{
ABSTRACT
}

Heterotopic ossification ( $\mathrm{HO}$ ) is a rare condition usually related to trauma or neurologic damage. It can affect all bone joints but is more frequent at the hip, particularly following total hip arthroplasty. This condition leads to significant impairment of life quality due to ectopic bone formation which causes functional loss. Here we describe two cases of patients presenting symptomatic HO classified as Brooker III following total hip replacement. One of them was treated prophylactically with external beam radiation, few hours before surgery for prosthesis revision. The other one, received radiotherapy as sole treatment with the intent of symptoms palliation. During the follow-up visits, the first patient didn't show any HO recurrence and had a significant improvement of his symptoms, namely the claudication; the last one achieved a significant relief of pain and showed an improvement on his functional capability. These cases show the potential benefit of radiotherapy as prophylaxis or as sole treatment of symptomatic HO.
\end{abstract}

Keywords: Heterotopic Ossification (HO); Hip Arthroplasty; External Beam Radiation Therapy (EBRT)

\section{Introduction}

Heterotopic ossification (HO) is an abnormal process that leads to the formation of lamellar bone in soft tissue, such as muscle, nerves or connective tissues [1-4]. There are some risk factors namely male gender, pre-existing ipsi- or contralateral HO, ankylosing spondylitis (Bekhterev's disease), disseminated idiopathic hyperostosis of the skeleton (Forestier disease), osteoarthritis and others [3,5]. Despite those, in general HO can be differentiated into three main categories: traumatic HO (eg. hip replacement, fracture, etc.), nontraumatic $\mathrm{HO}$ (mediated by genetic disorders like fibrodysplasia ossificans pregressiva), and neurologic HO (eg. myelitis; brain tumors) [1,6,7]. It has been postulated that $\mathrm{HO}$ pathogenesis is probably related to an inappropriate differentiation of mesenchymal stem cells into osteoblastic stem cells; however the definitive pathophysiologic causal factors remain uncertain. Although some authors identified some molecular and signaling pathways such as BMP-SMAD, Wnt/ $\beta$-catenin, Hedge hog and HIF- $1 \alpha$, which can be activated by different stimuli like tissue damage, inflammation, hypoxia, central nervous injury or others and ultimately led to $\mathrm{HO}$ [8].
Regarding to clinical presentation, $\mathrm{HO}$ is usually asymptomatic and detected only as an incidental finding on a radiograph. The most prevalent location is hip, for which there are four severity grades according to Brooker classification as follows:

a) Islands of bone within the soft tissues about the hip;

b) Bone spurs in the pelvis or the proximal end of the femur with at least $1 \mathrm{~cm}$ between the opposing bone surfaces;

c) Bone spurs from the pelvis or proximal end of the femur with less than $1 \mathrm{~cm}$ between opposing bone structures;

d) Radiographic ankylosis. The latter two are clinically symptomatic leading to motion impairment at the involved joint, pain and rarely to inflammatory signs like warmth, swelling and local erythema $[2,3,9]$. When $\mathrm{HO}$ causes symptoms it may decrease patient's quality of life in a meaningful manner. The two major treatment options are nonsteroidal anti-inflammatory drugs (NSAIDs) like indomethacin and radiotherapy, and most reports use them as prophylactic agents before or after surgery in high risk patients $[1,4,10,11]$. 
External beam radiation therapy (EBRT) is frequently delivered in a single fraction of 700-800 cGy within $4 \mathrm{~h}$ before surgery in the preoperative setting or within $72 \mathrm{~h}$ from the end of surgery when applied postoperatively. Studies didn't find any difference on efficacy of prophylactic radiotherapy given before or after surgery, and rates of $77-90 \%$ of complete response has been reported $[2,3,12,13]$. DEGRO practical guidelines recommends a singlefraction dose of 700-800 cGy or fractionated radiation with five fractions of 350 cGy for HO prophylaxis [3]. Data regarding the use of EBRT as sole treatment is more limited. Morcos et al. reported that low dose radiotherapy as an effective treatment in preventing the progression of $\mathrm{HO}$ in patients who unexpectedly develop significant HO following total hip arthroplasty, avoiding evolution to Brooker IV HO, with no patients presenting significant pain or limited hip motion after treatment [13]. Tao et al. also described a case successfully treated with EBRT, in which the patient achieved satisfactory pain relief and improvements in overall quality of life after a single fraction of 800 cGy [14]. In this article we describe two cases of HO where EBRT was used as prophylactic strategy before surgery (case 1) and as the only therapeutic approach for palliate HO symptoms (case 2).

\section{Case 1}

Male patient with 83 years of age, with history of total hip replacement 3 years ago, presenting HO Brooker III of the ipsilateral hip (Figure 1). He presented with pain and claudication. He underwent a single fraction of 750 cGy few hours before revision of the arthroplasty with substitution of the prosthesis on July of 2015. A 2D planning was done with AP-PA field toward the entire coxo-femoral joint (Figure 2). Treatment delivery was done on linear accelerator with photon energy of 18MV. Following treatment, patient experienced a significant improvement of his condition, with decrease on pain and regains the ability to do his daily activities by his own. On a radiograph taken 6 months after radiotherapy and surgery there are no signs of HO (Figure 3). Unfortunately he passed away three years later (July of 2018) of a mesothelioma diagnosed in 2017.

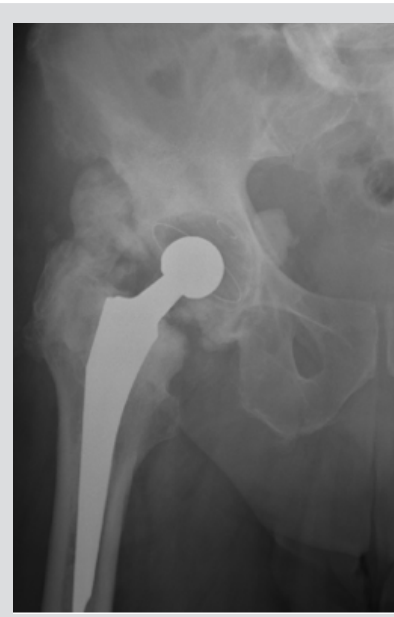

Figure 1: Right hip radiograph showing a Brooker III heterotopic ossification 3 years after total hip replacement.

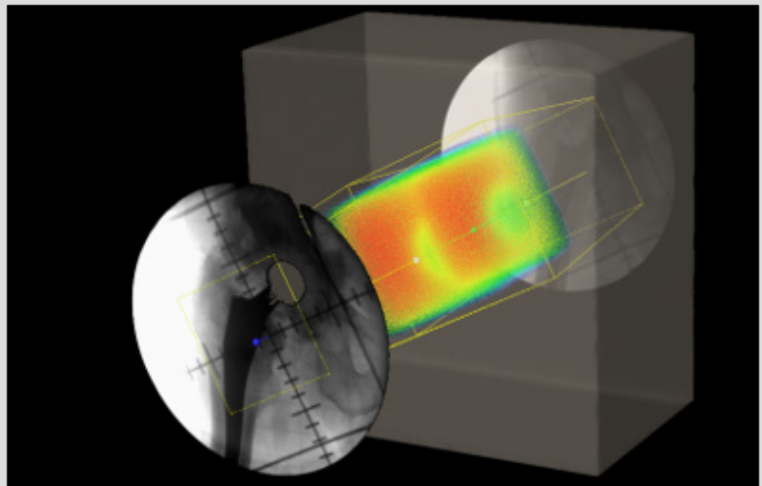

Figure 2: EBRT planning using a 2D technique with an AP-PA field.

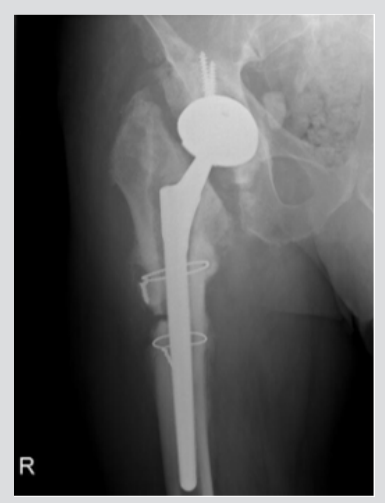

Figure 3: Control hip radiograph after radiotherapy and surgery showing no signs of $\mathrm{HO}$.

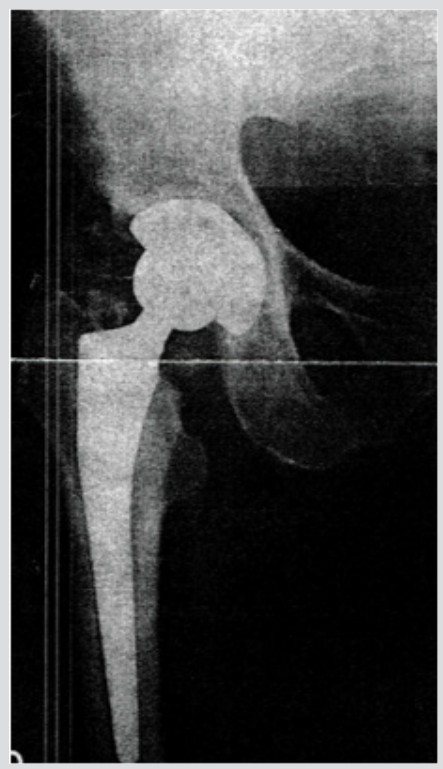

Figure 4: Control radiograph one month after right hip arthroplasty showing only mild signs of HO (Brooker I/ II).

\section{Case 2}

Male patient with 67 years of age, with previous total hip replacement 7 months ago. His comorbidities were osteoporosis, heart valve replacement and diabetes induced kidney injury. One 
month after surgery his radiograph showed smoldering signs of HO (Brooker I/II - Figure 4). Patient gradually developed pain, which got worse with time and decrease joint motion with progressively inability to flexion and elevation of the leg. On the control radiograph taken 5 months after surgery there were signs of HO Brooker III (Figure 5) and patient became more symptomatic with pain level 10 out a scale of 10 . He presented to our department on February 2019 with functional impairment, depression, pain and inability to perform his daily activities like driving. We performed a CT simulation on supine positioning, followed by a 3D conformal RT planning and treatment was delivered with a single fraction of 800 cGy on linear accelerator using photon energy of 18MV covering the entire joint (Figure 6). On the follow-up visit, one month after treatment, patient showed a decrease on his pain level (from 10 it decrease to 5 out a scale of 10) and regain some level of joint motion that allowed him to drive again and do some of his life activities.

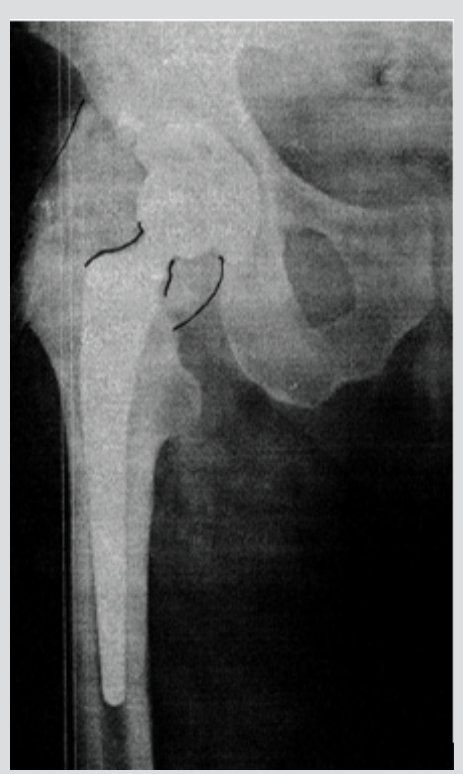

Figure 5: Brooker III HO found 5 months after surgery on hip radiograph.

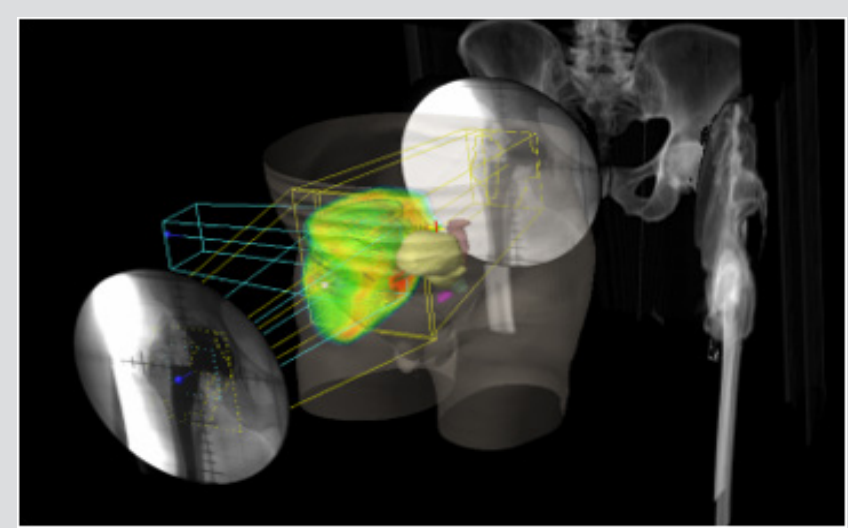

Figure 6: 3D conformal RT planning using 3 fields (AP, $\mathrm{PA}$ and lateral).

\section{Discussion}

HO is a well described condition, first reported in 1883 by Reidel, which leads to functional impairment and significant decrease in quality of life when symptomatic [15]. It may be caused by multiple factors such as traumatic injury, neurologic damage or burns that act as triggers to bone formation at ectopic sites. Most often it occurs at the hip after total arthroplasty, and there is a classification to grade the severity of HO based on radiograph findings. Typically Brooker III-IV HO are clinically relevant and symptomatic [1,9]. Both of our cases are classified as Brooker III of the hip and both developed following total hip arthroplasty. Treatment options include radiotherapy and NSAIDs or a combination of both. When performed EBRT tend to take place immediately before or after surgery as a prophylactic strategy to avoid recurrence of HO. Seegenschmied, randomized patients to receive EBRT either preoperatively $(<4 \mathrm{~h}$ before surgery) or postoperatively $(<72 \mathrm{~h}$ after surgery). Patients receiving preoperative treatment received 700 cGy in one fraction while patients receiving postoperative treatment received $17.500 \mathrm{cGy}$ in 5 fractions. Within the first group $(n=80)$ there were 19 treatment failures, and in the later $(n=81)$ there were 4 treatment failures.

The difference between the groups was statistically significant $(\mathrm{p}<0.05)$, but the authors stated that this difference was unapparent when comparing patients who preoperatively had Brooker grades $0-2$ [16]. On another randomized study, Gregoritch, compared the results of 122 patients that received 700-800 cGy in one fraction either before or after surgery and concluded that the preoperative and postoperative regimens are similar in efficacy [17]. Nowadays, prophylactic radiotherapy can be used before or after surgery, although some authors prefer the preoperative setting advocating that on postoperative setting there may be some obstacles related to transport difficulties and maneuver due to pain and the need to keep the mobilization of the joint minimized immediately after surgery [1].Regarding fractionation, several studies have been done in order to establish the optimal dose. Liu, randomized 147 high risk patients to receive either 400 cGy or 700 cGy on a single fraction one or two days after total hip arthroplasty. They found that 700 cGy was superior to 400 cGy in preventing HO formation following surgery [11] Similarly,

Healy, examined single dose irradiation with 700 cGy in comparison to $550 \mathrm{cGy}$ and concluded that the later dose is not sufficient for HO prophylaxis [18]. In an editorial about a systematic review published by Milakovic, Roos and Smith also defended that if EBRT is the chosen strategy for HO prophylaxis, treatment with a single 700-800 cGy fraction pre- or postoperatively seems optimal $[4,10]$. On the other hand, compared to fractionated treatments, single fraction have the advantages of causing less pain and risk of dislocation if given post-operatively or, in the pre-operative setting, RT can be completed a few hours immediately before 
surgery. One of the cases we report here, received a single fraction of 750 cGy immediately before surgery and there were none postoperative complications related to wound healing and the patient didn't experience local failure and a significant improvement of his life quality was achieved. In fact, according to DEGRO guidelines for the radiotherapy of non-malignant disorders, prophylactic radiotherapy is well tolerated and impaired wound healing has not been reported [3]. Pohl, also obtained excellent radiographic and functional outcomes after a single fraction of $700 \mathrm{cGy}$ administered before surgery [19].

Redda, retrospectively analyzed 30 patients after pre- or postoperative EBRT and obtained complete responses in 23 patients with excellent results in terms of joint mobility (the majority of patients received single fraction EBRT of 700 cGy) [2]. The use of radiotherapy as a prophylactic strategy is well established on the literature, and seems an efficient way of preventing the re-formation of ectopic bone, when delivered immediately before or after surgery. However its role on the treatment of this condition without surgery is less known. Morcos, studied the effect of late radiotherapy (delivered $>6$ weeks after surgery) on HO radiographically proven in 9 patients. They found that $89 \%$ of patients demonstrated no further progression in the amount of bone formed after EBRT treatment.13 This study contradicted the previously held belief that there were no role for radiotherapy in the management of HO more than $72 \mathrm{~h}$ after total hip arthroplasty. In fact several studies had demonstrated that EBRT after $72 \mathrm{~h}$ is ineffective for HO prophylaxis, but radiotherapy can be successfully used to prevent significant progression of the already forming HO [5,20,21].

Kantor, also achieved no further HO progression after EBRT treatment in patients with significant HO diagnosed more than 6 weeks after surgery [22]. Based on this postulated, we report a case of a patient treated only with single fraction of 800 cGy for a Brooker III hip HO, 6 months after surgery and he had a significant improvement of his pain and mobility, which ultimately led to an improved quality of life. Similarly, Tao et al. reported a case of a patient with extensive HO treated with a single fraction of 800 cGy and, at the 6-month follow-up visit, the patient also reported significant palliation of symptoms without signs of HO progression on radiographic images [14]. In terms of EBRT technique it seems that CT based EBRT allows more accurate delineation of the tissues and better clinical outcomes according to Mourad et al. [23]. In this article we reported two cases, one of them received 2D treatment and the other 3D conformational treatment. Both of them achieved relief of their symptoms and none experienced any immediate complications. However CT based EBRT is associated with additional cost which should be taken in consideration when treating these conditions.

It is also important to note that radiotherapy is not innocuous, and some authors manifested their concerns about second tumors risk and fertility issues, following radiotherapy. However, there have been no documented cases of radiation-induced tumors after EBRT for HO prevention [1]. This may be related, in part, to the relatively low dose used for treatment. On the other hand, these types of tumors are extremely rare and only arise after latencies of 10-30 years. In addition, the median age of patients presenting HO is 65 years, so, the risk of radiation-induced tumors is not relevant in this population.3 Regarding fertility issues, it is well known that doses as low as 1.200 cGy can confer permanent azoospermia and furthermore radiation-induced hereditary effects. Testicular shielding has been reported to reduce dose to the testis by about $54 \%$, thus this technique is strongly recommended in younger patients and all of those who manifest childbearing wishes [1,2]. On both of the cases that we described, testicular shielding was not used because the patients manifest no concerns regarding sperm count or infertility.

\section{Conclusion}

HO impairs quality of life causing several functional limitations. Radiotherapy can successfully be used to prevent its recurrence after surgery on the prophylactic setting, or to palliate symptoms on non-operative setting. One single fraction of 700-800 cGy can be delivered either before or after surgery, as well as the sole treatment of symptomatic HO, with satisfactory results in terms of pain control and joint motion.

\section{References}

1. Balboni T, Gobezie R, Mamon H (2006) Heterotopic Ossification: Pathophysiology, Clinical Features, and the Role of Radiotherapy for Prophylaxis. Int J Radiation Oncology Biol Phys 65(5): 1289-1299.

2. Redda M, Colle C, Bianco L (2018) Heterotopic ossifications: role of radiotherapy as prophylactic treatment. La radiologia medica 123: 463468.

3. Reinartz G, Eich H, Pohl F (2015) DEGRO practical guidelines for the radiotherapy of non-malignant disorders - Part IV Symptomatic functional disorders. Strahlenther Onkol 191: 295-302.

4. Milakovic M, Popovic M, Raman S (2015) Radiotherapy for the prophylaxis of heterotopic ossifications: A systematic review and metaanalysis of randomized controlled trials. Radiotherapy and Oncology 116: 4-9.

5. Koelbl O, Seufert J, Pohl F (2003) Preoperative irradiation for prevention of heterotopic ossification following prosthetic total hip replacement results of a prospective study in 462 hips. Strahlenther Onkol 179: 767773.

6. Stoikovic JP, Bonfiglio M, Paul WD (1955) Myositis ossificans complicating poliomyelitis. Arch Phys Med Rehabil 36: 236-243.

7. Roberts PH (1968) Heterotopic ossification complicating paralysis of intracranial origin. J Bone Joint Surg Am 50: 70-77.

8. Legosz P, Drela K, Pulik L (2018) Challenges of heterotopic ossification - Molecular background and current treatment strategies. Clin Exp Pharmacol Physiol 45: 1229-1235.

9. Brooker AF, Bowerman JW, Robinson RA (1973) Ectopic ossification following total hip replacement. Incidence and a method of classification. J Bone Joint Surg Am 55: 1629-1632.

10. Roos D, Smith JG (2015) Radiotherapy for the prophylaxis of heterotopic ossification: A single 7-8Gy fraction seems optimal (editorial). Radiotherapy and Oncology 116: 1-3. 
11. Liu JZ, Frish NB, Barden RM (2017) Heteroptopic Ossification Prophylaxis After Toral Hip Arthroplasty: Randomized Trial of 400 vs 700 cGy. The Journal of Arthroplasty 32: 1328-1334.

12. Lo TC, Healy WL, Covall DJ (1988) Heterotopic bone formation after hip surgery: Prevention with single-dose postoperative hip irradiation. Radiology 168: 851-854.

13. Morcos M, Smith K, Tanzer M (2018) The effect of late radiotherapy on the progression of heterotopic ossification following total hip arthroplasty. European Journal of Orthopaedic Surgery \& Traumatology 28: 1125-1131.

14. Tao MJ, Probyn L, Poon M (2017) Symptomatic palliation with radiotherapy in extensive heterotopic ossifications. Ann Palliat Med 6(2): S260-S263.

15. Stoltny T, Koczy B, Wawrzynek W (2007) Heterotopic ossification in patients after total hip replacement. Ortopedia Traumatologia Rehabiticja 3(6): 267-272.

16. Seegenschmiedt MH, Keilholz L, Martus P (1997) Prevention of heterotopic ossification about the hip: Final results of two randomized trials in 410 patients using either preoperative or postoperative radiation therapy. Int J Radiat Oncol Biol Phys 39: 161-171.

17. Gregoritch SJ, Chadha M, Pelligrini VD (1994) Randomized trial comparing preoperative versus postoperative irradiation for prevention of heterotopic ossification following prosthetic total hip replacement: Preliminary results. Int J Radiat Oncol Biol Phys 30: 55-62.

\section{ISSN: 2574-1241}

DOI: 10.26717/BJSTR.2019.16.002907

Andreia Ponte. Biomed J Sci \& Tech Res

This work is licensed under Creative

Commons Attribution 4.0 License

Submission Link: https://biomedres.us/submit-manuscript.php
18. Healy WL, Lo TC, DeSimone AA (1995) Single-dose irradiation for the prevention of heterotopic ossification after total hip arthroplasty. A comparison of doses of five hundred and fifty and seven hundred centigray. J Bone Joint Surg Am 77: 590-595.

19. Pohl F, Seufert J, Tauscher A, Lehmann H, Springorum HW, et al. (2005) The influence of heterotopic ossification on functional status of hip joint following total hip arthroplasty. Strahlenther Onkol 181(8): 529-533.

20. Board TN, Karva A, Board RE, Gambhir AK, Porter ML, et al. (2007) The prophylaxis and treatment of heterotopic ossification following lower limb arthroplasty. J Bone Joint Surg Br 89(4): 434-440.

21. Pellegrini VD Jr, Konski AA, Gastel JA, Rubin P, Evarts CM, et al. (1992) Prevention of heterotopic ossification with irradiation after total hip arthroplasty. Radiation therapy with a single dose of eight hundred centigray administered to a limited field. J Bone Joint Surg Am 74(2): 186-200.

22. Kantor SR, Cummins J, Tanzer M (2005) Complications after total hip arthroplasty: heterotopic ossification. Semin Arthroplasty 16(2): 10113.

23. Mourad WF, Packianathan S, Ma JK (2013) Computerized tomographybased radiotherapy improves heterotopic ossification outcomes. Bone 57: 132-136.

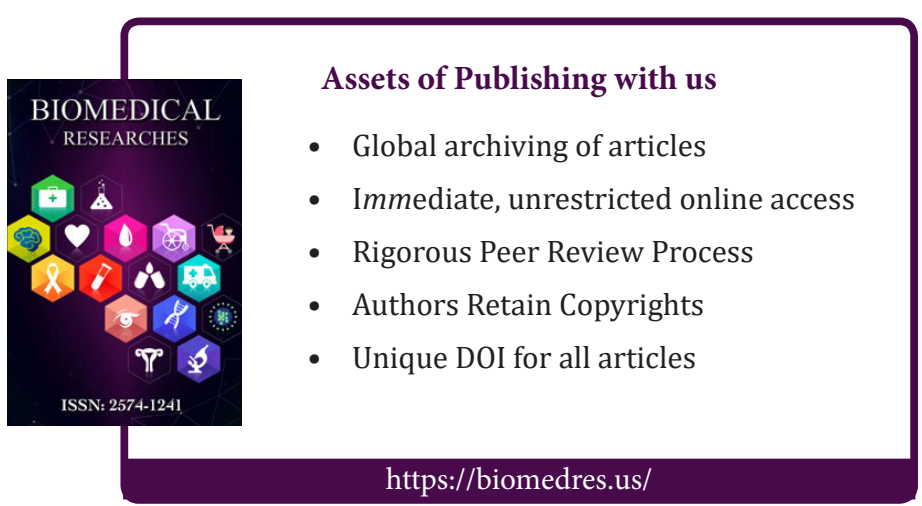

